\title{
Proposta de uma Plataforma Crowdsourcing para Apoio à Tomada de Decisão Médica
}

\author{
Álisson Oliveira dos Santos ${ }^{1}$, Leonardo Cançado Monteiro Savassi², Alexandre \\ Sztajnberg ${ }^{1}$ \\ ${ }^{1}$ Centro Biomédico - Mestrado Profissional em Telemedicina e Telessaúde - \\ Universidade do Estado do Rio de Janeiro (UERJ) \\ ${ }^{2}$ Escola de Medicina - Universidade Federal de Ouro Preto (UFOP) \\ alisson.ufop@gmail.com, savassi@medicina.ufop.br, alexszt@ime.uerj.br
}

\begin{abstract}
Medical summaries made available as digital tools have been increasingly used to support medical decision making. Although widely available, such tools present barriers to its adoption, among them the high cost. This paper presents the proposal of a medical summaries platform using the collaborative model, crowdsourcing, as a way to overcome the barriers of traditional tools, where the users themselves are responsible to add and maintain abstracts. The development of the platform, which includes web and mobile applications, is underway.
\end{abstract}

Resumo. Os sumários médicos disponibilizados na forma de ferramentas digitais têm sido cada vez mais usados para apoiar a tomada de decisão médica. Apesar de amplamente disponiveis, tais ferramentas apresentam barreiras para sua adoção, entre elas o custo elevado. Este trabalho apresenta a proposta de uma plataforma de sumários médicos utilizando o modelo colaborativo, crowdsourcing, como forma de superar as barreiras das ferramentas tradicionais, onde os próprios usuários são responsáveis por adicionar e manter os resumos. O desenvolvimento da plataforma, que inclui aplicativos web e mobile encontra-se em andamento.

\section{Introdução}

A tomada de decisão médica é historicamente apoiada por consulta a materiais referenciados, que auxiliam desde a verificação da dose mais adequada de um medicamento, critérios diagnósticos de uma determinada doença, até mesmo o melhor tratamento a ser prescrito. Esse processo, por sua vez, tem se modificado nos últimos anos na medida em que médicos que outrora utilizavam materiais impressos, como livros e revistas científicas, contam agora com diversas soluções digitais com o mesmo resultado [Ventola, 2014].

A oferta de soluções digitais vem aumentando de forma considerável nos últimos anos. Entre elas, podemos citar os e-books - versões digitalizadas de livros -, os sistemas de apoio à decisão clínica - que utilizam inteligência computacional para inferência de condições de saúde ou tratamento mais adequado a partir de dados clínicos do paciente - e os sumários médicos, que são compilados sobre doenças e condições 
médicas, apresentando informações robustas e baseadas nas melhores evidências científicas disponíveis [Santos, 2010].

Os sumários médicos estão entre as ferramentas mais utilizadas pelos profissionais, em especial após a popularização de dispositivos móveis, como tablets e smartphones. Com eles, os profissionais passaram a ter na "palma da mão" informações atualizadas sobre a definição, diagnóstico e tratamento de doenças, condições de saúde e outros problemas de relevância médica. Aplicações e portais de sumários médicos tem como base a Medicina Baseada em Evidências (MBE), conceito que se refere à aplicação do melhor conhecimento científico, levando em consideração a experiência clínica e o contexto dos pacientes [Lopes, 2000]. Estas aplicações tornaram-se referência de suporte para consulta, tomada de decisão e para educação médica, visto que fornecem conteúdo sintetizado e de acordo com estudos mais recentes. Entre os exemplos populares destas aplicações estão o UpToDate (www.uptodate.com/), Dynamed (www.dynamed.com/) e BMJ Best Practice (bestpractice.bmj.com/).

As plataformas de sumários médicos disponíveis atualmente enfrentam desafios referentes à sua implantação e manutenção, representando barreiras à sua ampla adoção. A principal delas é o custo. Manter atualizado o acervo de resumos tem um custo significativo, pois envolve grande quantidade de profissionais em diversas especialidades. Outro desafio relaciona-se ao tempo em que um sumário de alta qualidade leva para ser produzido, visto que este resulta da compilação de muitos de estudos diariamente publicados.

Em um contexto com menos restrições quanto à qualidade das informações, aplicações como a Wikipédia adotam o modelo de crowdsourcing, ou desenvolvimento colaborativo pelos usuários, os quais compartilham conhecimento para resolução de problemas [Ermoshina, 2017]. A construção do conteúdo da Wikipédia, por exemplo, se dá de forma compartilhada e livre. Este modelo parece promissor para o caso de sumários médicos. A proposta envolveria um sistema, onde profissionais de saúde contribuiriam solidariamente e gratuitamente para manter um repositório de sumários de qualidade, que poderia ser consultado gratuitamente. Dessa forma, ao invés de simplesmente utilizar o conteúdo desenvolvido por uma plataforma paga, ou disponibilizado por organizações de classe, dá-se ao usuário a oportunidade de ser o protagonista de criação e atualização da plataforma de sumários, bem como pela sua qualidade e regulação.

Este artigo apresenta a proposta de uma plataforma crowdsourcing de apoio à tomada de decisão clínica, baseada em sumários médicos. A utilização desse modelo pode apresentar-se como solução ao desafio do custo e de tempo, caso haja a adesão de um grupo significativo de profissionais. Entretanto, este modelo impõe um novo desafio: garantir a qualidade dos sumários inseridos. Por se tratar de conteúdo para tomada de decisão ou a educação médica, a validade e confiabilidade das informações inseridas na plataforma são cruciais.

Portanto, como parte da solução, propõe-se também tratar o problema da qualidade, a partir de ferramentas de validação do conteúdo criado, e oferecendo a plataforma inicialmente a um grupo restrito, dentro dos Programas de Residência Médica (PRM). Dentre as práticas destes programas há habitualmente a discussão e construção de evidências, tendo em vista se tratar de um processo de formação de 
médicos com treinamento em serviço, carga horária densa, e discussão ampla da melhor evidência científica para a tomada de decisões a cada paciente atendido.

O restante do texto está organizado da seguinte forma: a Seção 2 traz uma revisão de trabalhos relacionados; a Seção 3 discute o modelo proposto para plataforma de sumários; a Seção 4 apresenta o estágio atual de desenvolvimento da proposta e a Seção 5 apresenta algumas discussões. A Seção 6 conclui o artigo.

\section{Trabalhos Relacionados}

Para verificar-se a precedência da construção da plataforma de sumários, uma revisão da literatura foi realizada nas bases Pubmed, Cochrane e Scielo. Usando os termos "point-of-care AND summaries", chegou-se a um total de 132 artigos, os quais foram capturados em janeiro de 2016. Foram excluídos 120 artigos que não tratavam sobre sumários médicos para tomada de decisão, resultando em 12 estudos incluídos nos critérios estabelecidos. Desses, não foram encontrados estudos que abordassem sumários médicos colaborativos, prevalecendo as ferramentas tradicionais.

Para analisar os precedentes do uso do crowdsourcing como modelo para a construção e difusão de conhecimento em saúde baseado em evidências, uma revisão sistematizada da literatura foi realizada, buscando-se os termos "crowdsourcing" e "evidence-based medicine" nas bases de dados PubMed, Scielo e Google Acadêmico. A partir dos 433 artigos, os critérios de inclusão foram: artigos que apresentavam o crowdsourcing para compilação de evidências; artigos que comparavam o crowdsourcing com o formato convencional. Os critérios de exclusão, por sua vez foram: artigos que não tratavam da área médica; artigos que não relacionavam o crowdsourcing no contexto da medicina baseada em evidências. Com esse filtro, apenas dois artigos foram encontrados.

Mortensen et. al. (2017), utilizaram o crowdsourcing para conduzir revisões sistemáticas, comparando os resultados com as mesmas revisões feitas por especialistas treinados. Como resultados, foi possível identificar $99 \%$ dos estudos em comparação com as revisões tradicionais, apresentando um custo cerca de $88 \%$ menor.

Wallace et. al. (2017), conduziram um estudo no qual uma plataforma colaborativa visava identificar Ensaios Clínicos Randomizados (ECR) verdadeiros em detrimento de estudos que não se tratavam de ECR. Foi possível identificar a partir do modelo proposto, 95 a 99\% dos estudos de alta qualidade, com um esforço calculado $80 \%$ menor que a identificação feita por especialistas.

\section{A Plataforma Proposta}

A Figura 1 apresenta a estrutura geral da plataforma. Um usuário, médico ou estudante de medicina, previamente cadastrado, pode acessar o conteúdo através de uma interface, bem como criar novos conteúdos, editar informações pré-existentes e atualizar aqueles conteúdos construídos por outros usuários. A plataforma organiza o controle de acesso do usuário, bem como seu cadastro e os mecanismos de avaliação e de reputação por participação. As informações da plataforma, como os sumários desenvolvidos e a base de usuários são armazenadas em uma base de dados. 


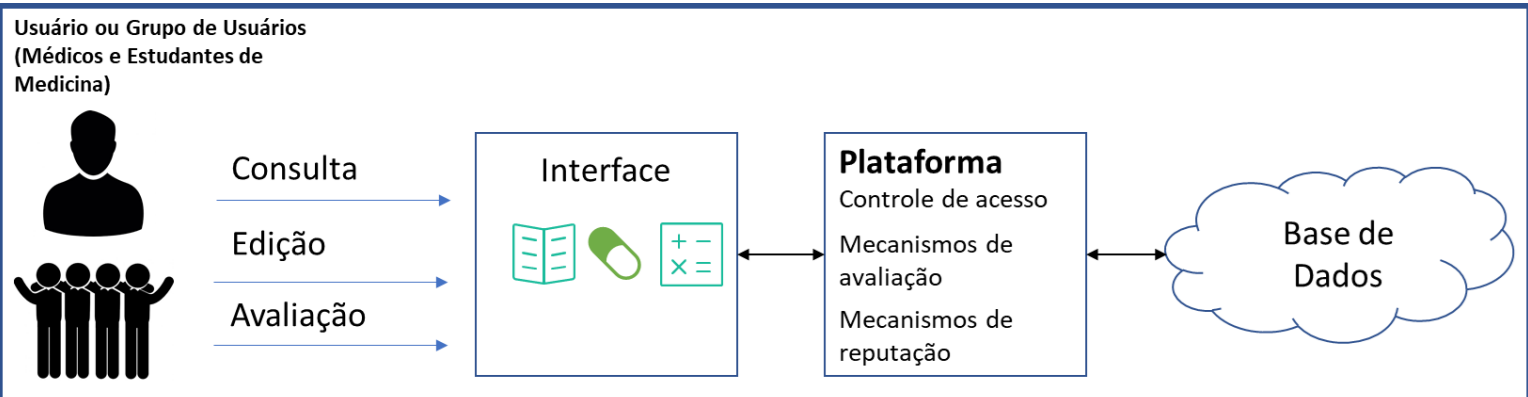

Figura 1. Estrutura da plataforma

Módulos. A plataforma é planejada a partir de três módulos principais:

- tópicos: sumários de doenças e condições médicas, com definição, etiologia, epidemiologia, diagnóstico, tratamento, entre outras informações;

- medicamentos: informações sobre dose, efeitos colaterais, farmacologia, etc.;

- calculadoras: utilizadas para obter dados antropométricos, índices clínicos, predição de gravidade de certas condições, dose de infusão de certas drogas e várias outras situações que demandam cálculos na prática profissional.

Colaboração. A partir da participação na construção dos conteúdos, seja criando ou editando informações, o nome dos autores é incluído, creditando a colaboração. Além disso, podem-se criar grupos de colaboradores, ampliando a rede participativa.

Avaliação. Além de criar e editar conteúdos, os usuários serão responsáveis por avaliar cada assunto inserido, seja ele um tópico, uma calculadora ou um medicamento. Ele poderá atribuir uma nota de 0 a 5 , sendo a média das avaliações apresentada na estrutura da informação.

Valide e Confiabilidade. A solução proposta para a validade e confiabilidade dos sumários é baseada em uma avaliação multiparte: (i) dos usuários, a partir das notas a cada conteúdo criado e, (ii) dos tutores e coordenadores de PRM em Medicina de Família e Comunidade (MFC), que analisarão uma amostra do conteúdo a partir de ferramentas de gradação de evidências, verificando a qualidade do conteúdo.

Recompensa. A partir da participação na plataforma os usuários receberão pontos. A partir desses pontos, pretende-se premiar os usuários e/ou grupos de usuários mais ativos, inclusive com possibilidade de prêmios reais, como descontos em congressos ou pontuação para provas de título e residência. Espera-se que o ambiente de competição gerado, principalmente entre PRM distintos, estimule a participação.

\section{Protótipo e Estágio Atual}

A construção da plataforma está em desenvolvimento a partir de ferramentas web (JavaScript, HTML5 e CSS), utilizando o framework Angular ${ }^{\circledR}$. A persistência de informações, em especial armazenamento de informações, cadastro de usuários e acesso, tem o suporte da ferramenta Firebase ${ }^{\circledR}$. A plataforma poderá ser acessada a partir da web e por aplicativo para tablets e smartphones, pelos sistemas operacionais Android e $i O S$. O modelo de dados inicial é apresentado na Figura 2, com os principais elementos 
e suas relações. A forma de relacionar uma avaliação (boa ou ruim) e a reputação do usuário ou seu grupo ainda está sendo estudada.

A Figura 3 apresenta algumas telas da aplicação móvel de acesso à plataforma. Outros módulos visuais não exibidos aqui também estão prototipados. A estrutura básica de interface já se encontra desenvolvida, já é possível realizar cadastro de usuário e login, bem como criar tópicos e editá-los. Demais usuários da plataforma também já podem acessar os tópicos criados e acrescentar ou modificar o que desejarem.

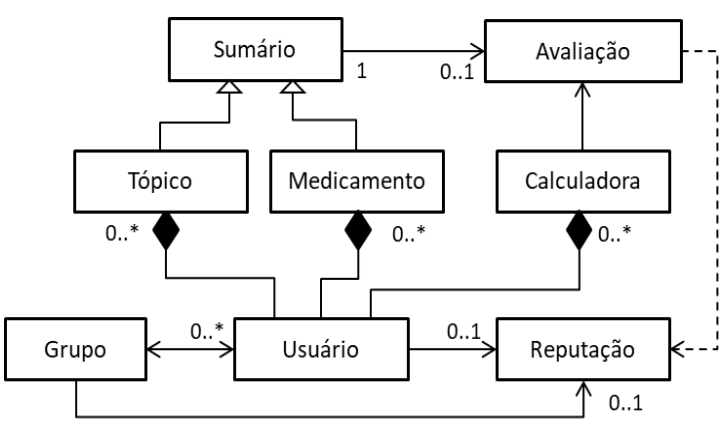

Figura 2. Modelo de dados

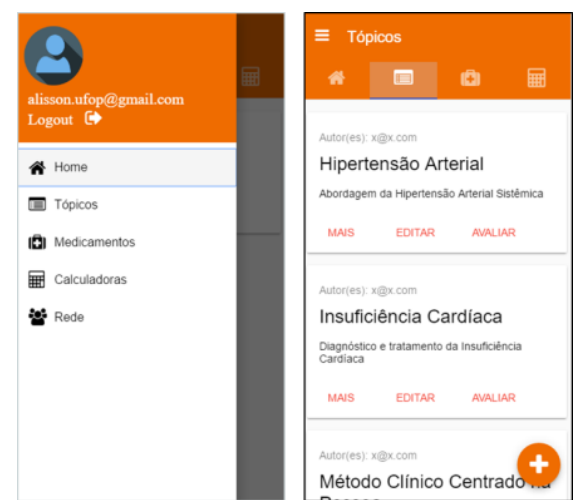

Figura 3. Interface Android do protótipo

\section{Discussão}

A utilização de ferramentas de desenvolvimento web já consolidadas tem se mostrado uma escolha acertada. Mecanismos de autenticação de usuário e a integração com o Firebase são simplificados, bem como a geração de versões para plataformas distintas, facilitada com o uso do Angular. Os testes de operação com os protótipos desenvolvidos têm sido satisfatórios.

As próximas etapas de desenvolvimento incluem as rotinas de interfaces para avaliação dos tópicos pelos usuários e o sistema de pontuação por participação. Também será introduzido um sistema de verificação de versão, ou seja, poder voltar atrás caso um tópico seja totalmente modificado. Essa funcionalidade está próxima de ser operacionalizada. A possibilidade de inserção de conteúdos como medicamentos e calculadoras médicas serão implantadas na última etapa.

A avaliação e validação dos conteúdos terá atenção na sequência. Com relação à avaliação por notas pelos usuários, problemas relacionados a inconsistências estão em estudo. Por exemplo, caso algum usuário modifique um conteúdo, as notas atribuídas previamente teriam que ser descartadas. Portanto, formas de manter ou preservar parte da avaliação de conteúdo ainda estão em estudo.

Por sua vez, a avaliação de uma amostra do conteúdo por tutores e coordenadores dos PRM em MFC também está em discussão. Uma ferramenta de avaliação de evidências já consolidada deverá ser escolhida, levando em consideração a sua confiabilidade e facilidade na aplicação.

Além da implementação, planejamos validar a proposta e a plataforma. A partir disso, pretendemos estudar e aplicar testes e ferramentas de avaliação de usabilidade e de satisfação, servindo como base para atualizações e adaptações da plataforma. 


\section{Conclusão}

Observa-se que o uso do crowdsourcing pode representar um caminho a ser seguido no que se refere à consolidação de uma plataforma de sumários médicos capaz de superar as principais barreiras de operacionalização dos modelos tradicionais.

Os próximos passos envolverão a finalização do desenvolvimento da plataforma, os testes piloto e as avaliações propostas. Espera-se contar com a adesão de usuários residentes, tutores, preceptores e coordenadores dos PRM, a qual será definidora para o alcance e sucesso da plataforma e para o fortalecimento das tomadas de decisão baseadas em evidências na área médica. Além disso, os resultados iniciais de validação serão fundamentais para planejar a abertura da plataforma para o público geral, não mais restringindo aos programas de residência.

O projeto está sendo desenvolvido no contexto do Mestrado Profissional em Telemedicina e Telessaúde da Universidade do Estado do Rio de Janeiro (UERJ). As etapas de desenvolvimento e validação estão sendo realizadas em colaboração com a Universidade Federal de Ouro Preto (UFOP) e de PRM em MFC.

\section{Referências}

Ermoshina, K. (2016) Is There an App for Everything? Potentials and Limits of Civic Hacking. Observatorio $\left(O B S^{*}\right), 10$ (Especial), 116-140.

Lopes, A. A. (2000). Medicina Baseada em Evidências: a arte de aplicar o conhecimento científico na prática clínica. Revista da Associação Médica Brasileira, 46(3), 285288.

Mohammed, M. A., Bright, A. S., Apostolic, C., et al (2017) Mobile-Based Medical Health Application - Medi-Chat App. International Journal of Scientific \& Technology Research, Si, v. 6, n. 05, p.70-76.

Mortensen, M. L., Adam, G. P., Trikalinos, et al. (2017) An exploration of crowdsourcing citation screening for systematic reviews. Research Synthesis Methods, v. 8, n. 3, p.366-386.

Santos, A. N. e Mercado, L. P. L. (2010) Arquivamento e comunicação de imagens radiológicas na formação médica online. Revista Brasileira de Educação Médica, 34(4), 525-534.

Ventola, C. L. (2014) Mobile Devices and Apps for Health Care Professionals: Uses and Benefits. Pharmacy And Therapeutics, v. 39, n. 5, p.356-364.

Wallace, B. C., Noel-Storr, A., Marshall, I. J., et al (2017) Identifying reports of randomized controlled trials (RCTs) via a hybrid machine learning and crowdsourcing approach. Journal of The American Medical Informatics Association, v. 24, n. 6, p. $1165-1168$. 